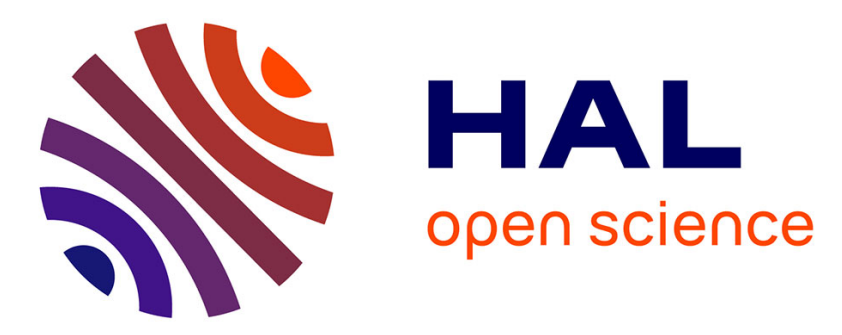

\title{
Scattering of a magnetostatic surface spin wave from a one-dimensional step potential in a ferromagnetic film
}

\author{
Arjun Balaji, Mikhail Kostylev, Matthieu Bailleul
}

\section{To cite this version:}

Arjun Balaji, Mikhail Kostylev, Matthieu Bailleul. Scattering of a magnetostatic surface spin wave from a one-dimensional step potential in a ferromagnetic film. Journal of Applied Physics, 2019, 125 (16), pp.163903. 10.1063/1.5091806 . hal-02415811

\section{HAL Id: hal-02415811 \\ https://hal.science/hal-02415811}

Submitted on 17 Dec 2019

HAL is a multi-disciplinary open access archive for the deposit and dissemination of scientific research documents, whether they are published or not. The documents may come from teaching and research institutions in France or abroad, or from public or private research centers.
L'archive ouverte pluridisciplinaire HAL, est destinée au dépôt et à la diffusion de documents scientifiques de niveau recherche, publiés ou non, émanant des établissements d'enseignement et de recherche français ou étrangers, des laboratoires publics ou privés. 


\section{Scattering of a magnetostatic surface spin wave from a one-dimensional step potential in a ferromagnetic film $\oplus$}

Cite as: J. Appl. Phys. 125, 163903 (2019); https://doi.org/10.1063/1.5091806

Submitted: 05 February 2019 . Accepted: 04 April 2019. Published Online: 29 April 2019

Arjun Balaji (D), Mikhail Kostylev (D), and Matthieu Bailleul (D)

\section{COLLECTIONS}

EP This paper was selected as an Editor's Pick
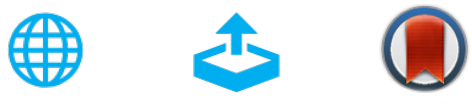

\section{ARTICLES YOU MAY BE INTERESTED IN}

Accurate manipulation of optogenetic proteins with wavelength tunable femtosecond laser system

Journal of Applied Physics 125, 163105 (2019); https://doi.org/10.1063/1.5084197

Myths about new ultrahard phases: Why materials that are significantly superior to diamond in elastic moduli and hardness are impossible

Journal of Applied Physics 125, 130901 (2019); https://doi.org/10.1063/1.5082739

Covalent attachment of streptavidin to two dimensional magnetic nanocomposite enhances surface enhancement Raman spectroscopic signal

Journal of Applied Physics 125, 164902 (2019); https://doi.org/10.1063/1.5079607

\section{ri AlluXa YOUROPTICAL COATING PARTNER}




\title{
Scattering of a magnetostatic surface spin wave from a one-dimensional step potential in a ferromagnetic film
}

Cite as: J. Appl. Phys. 125, 163903 (2019); doi: 10.1063/1.5091806

Submitted: 5 February 2019 - Accepted: 4 April 2019.

Published Online: 29 April 2019

Arjun Balaji, ${ }^{1}$ (D) Mikhail Kostylev, ${ }^{7, a)}$ (D) and Matthieu Bailleul ${ }^{2}$ (D)

\begin{abstract}
AFFILIATIONS
${ }^{7}$ Department of Physics and Astrophysics, The University of Western Australia, Crawley, 6009 WA, Australia

${ }^{2}$ Université de Strasbourg, CNRS, Institut de Physique et Chimie des Matériaux de Strasbourg, UMR 7504, 67034 Strasbourg, France
\end{abstract}

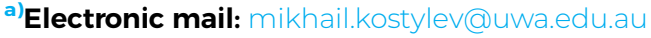

\begin{abstract}
As the research on magnetostatic surface spin waves (MSSWs) gains momentum, a concrete understanding of how these waves behave at interfaces is still undeveloped. In this work, we theoretically investigate how a MSSW traveling in a ferromagnetic film scatters across a sharp step of magnetic parameters in the film such as the applied field. An integral equation is derived, which describes the scattering process. We solve this equation both directly numerically and using a second Born approximation. We find that scattering produces partial reflection and partial transmission of the wave through the step. A nontrivial behavior of the amplitude of the transmitted wave as a function of the step size is observed in the calculation and analyzed.
\end{abstract}

\section{Published under license by AIP Publishing. https://doi.org/10.1063/1.5091806}

\section{INTRODUCTION}

Scattering of spin waves from localized inhomogeneities in ferromagnetic films has been studied extensively. ${ }^{1-18}$ It has been shown that a single scatterer, for instance, a thin wire sitting on top of a ferromagnetic film that carries a DC current, creates a strongly localized magnetic field from which an incident plane backward volume magnetostatic spin wave (BVMSW) can efficiently scatter. $^{12,13}$

Two regimes were identified in this case; one is tunneling, when the area of field nonuniformity acts as an energy barrier for spin waves (magnons), and the only way for a wave to cross the nonuniformity is by tunneling. ${ }^{13}$ The energy barrier is formed because the spin wave spectrum in an in-plane magnetized ferromagnetic film is characterized by an energy gap due to Zeeman and shape anisotropy contributions to spin wave dispersion. If the frequency of a wave incident onto the nonuniformity falls into the energy gap for the barrier, one deals with the tunneling regime. In the case of BVMSW, this regime is implemented when the Oersted field of the current in the wire is antialigned to the uniform magnetic field applied to the film, thus creating a "valley" of the total magnetic field in the vicinity of the wire.
The second regime is scattering. In this case, propagation of spin waves inside an inhomogeneity is allowed, but with a different wave number with respect to the wave incident onto the inhomogeneity. ${ }^{14}$ In the same example of a wire carrying a DC current, this regime is implemented by reversing the direction of the current. A "hump" of the total applied field is formed in this case, and a BVMSW in the hump must have a larger wave number for the same frequency compared to elsewhere. For the localized inhomogeneity, the scattering has a resonance character, a wave bounces several times between the boundaries of the nonuniformity before escaping it. These types of oscillations that are relatively strongly coupled to the environment are called quasinormal modes. ${ }^{19}$ Formation of the quasinormal modes inside the field hump creates a very strong suppression of the amplitude of the wave transmitted through the inhomogeneity for some frequencies. The loss inserted by the hump is even bigger than in the tunneling regime for the same magnitude of the current. Previous work shows that if the magnitude of the field's hump is controlled electrically, the scattering geometry represents a very efficient spin wave transistor. ${ }^{14}$ Its operation in the on/off regime allows us to build logic circuits based on traveling spin waves. ${ }^{20,21}$ 
It was later demonstrated that scattering from multiple parallel wires carrying a current allows one to build a dynamic magnonic crystal. ${ }^{17,18}$ Here, one has to mention that creating a hump or a valley of an applied field is not the only method to produce a scatterer. Etching grooves in a film ${ }^{1-7}$ or depositing nonmagnetic metal stripes on top of it leads to the same effect. ${ }^{8}$ Furthermore, locally etching a film away completely creates a mechanical gap through which a spin wave can tunnel due to its long-ranging magnetic dipole field. ${ }^{15}$

Obviously, contrary to the barrier created by a nonuniformity of a magnetic field, etching materials away can create a very sharp step of material parameters, as does deposition of metals on the film surface. The latter geometry has been treated theoretically before. ${ }^{9}$ There are other methods which may also create a sharp step of material parameters. For instance, a step of film saturation magnetization can be produced by ion implantation ${ }^{22}$ or by deposition of two different materials in lateral contact. ${ }^{23}$ Sharp steps of effective fields such as the perpendicular magnetic anisotropy, Dzyaloshiskii-Moriya interaction, ${ }^{24}$ or exchange bias ${ }^{25}$ can also be created. This can be done by interfacing a ferromagnetic layer with a nonmagnetic or an antiferromagnetic layer. The effective field will vary on the length scale of the exchange length for the ferromagnetic material. For instance, the exchange length for Permalloy is on the order of $10 \mathrm{~nm}$. This length scale is much smaller than the one for the spin wave dynamics in most experiments on traveling spin waves. With this in view, in this work, we consider theoretically scattering a magnetostatic surface spin wave (MSSW) from an infinitely sharp step of material parameters, such as the effective field. The area behind the step is considered to be of an infinite length. Thus, there is no second boundary of the nonuniformity, contrary to the cases of localized inhomogeneities studied before. $^{1-18}$ This allows us to produce quasianalytical expressions for the transmission and reflection coefficients for the step. We utilize a similar integration technique found in prior work that involves a Green's function of spin wave excitation. ${ }^{14}$ Note that smooth (but narrow) potential barriers have been considered before and compared with sharp barriers of the same length. ${ }^{14}$ Much stronger variation of transmission and reflection with the height of the potential barrier was observed in that work for the barriers with the sharp edges (Fig. 5 in that paper).

The paper is organized as follows: Sec. II describes the theory, and Sec. III discusses the effect of a step characterized by the applied field. Section IV describes the results, starting with a discussion of the main characteristics of the scattered wave (Sec. IV A), proceeding with the derivation of the transmission coefficient (Sec. IV B), and with the treatment of the Born approximation (Sec. IV C). The conclusions are presented in Sec. V.

\section{THEORY}

\section{A. Equations of motion}

Consider Fig. 1(a), a film perpendicular to the $z$-direction made of a ferromagnetic material extended in the $x y$ plane with a thickness $L$ in the $z$-direction. A homogeneous external magnetic field of strength $H_{\text {ext }}$ is applied such that $\vec{H}_{\text {ext }}=H_{\text {ext }} \hat{\mathbf{y}}$ and the static magnetization vector of strength $M_{s}$ is parallel to the external field, $\vec{M}_{s}=M_{s} \hat{\mathbf{y}}$. We investigate MSSWs that travel in the positive

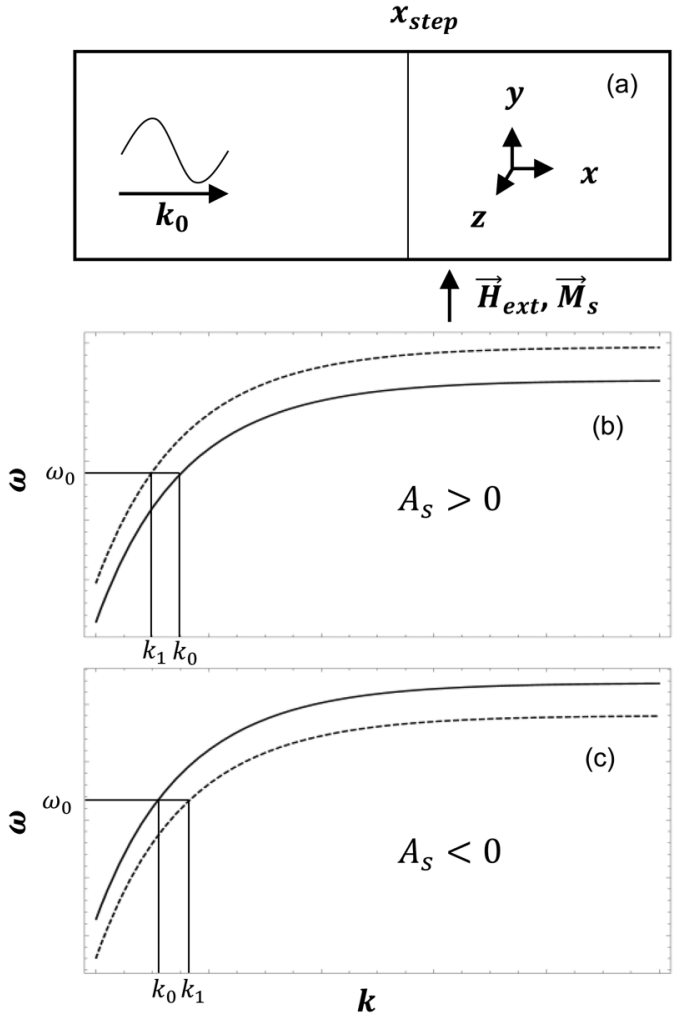

FIG. 1. (a) $2 \mathrm{D}$ top-down view of the geometry. (b) MSSW dispersion relation shift for the $A_{s}>0$ regime. (c) MSSW dispersion relation shift for the $A_{s}<0$ regime. Solid line is the initial dispersion relation in region 0 , and the dashed line is the dispersion relation after the step in region 1. Note that this plot is for illustrative purposes and thus the values are omitted.

$x$-direction. The spin-wave wave number is $k$ and its frequency is $\omega$. A spin wave with a specific positive wave number $k_{0}$ is incident on a step potential of amplitude $A_{s}$ from the left and along the $x$-axis as described in Fig. 1(a).

The incident wave, with positive wave number $k_{0}$, is generated by a source of frequency $\omega$ located at $x=0$. The wave propagates to the right and reaches a step potential at $x=x_{\text {step }}$ [Fig. 1(a)], where we assume that $x_{\text {step }}$ is large enough to allow us to only consider far-field effects at $x \geq x_{\text {step }}$.

To arrive at the magnetization dynamics for the MSSW, we solve the Landau-Lifshitz equation in the exchange free approximation [Eq. (1)] in the very large wavelength limit and Maxwell's equations in the magnetostatic limit [Eqs. (2) and (3)]. Furthermore, we assume that the film thickness is small enough to satisfy $k L \ll 1$, and the dynamic magnetization and effective magnetic field are averaged over the film thickness. ${ }^{26}$

Here, magnetocrystalline anisotropy is neglected, which is valid for soft ferromagnetic materials such as Permalloy. The exchange interaction is also neglected, which is expected to hold good for small enough wave vectors. Note that all variables depend on space and time $(x, t)$, but this will be left out to tidy up the equations 
unless explicitly stated otherwise,

$$
\begin{gathered}
\frac{\partial \vec{M}}{\partial t}=-|\gamma|\left(\vec{M} \times \vec{H}_{e f f}\right), \\
\nabla \times \vec{h}_{d}=0, \\
\nabla \cdot\left(\vec{h}_{d}+4 \pi \vec{m}\right)=0,
\end{gathered}
$$

where $\vec{M}$ is the total magnetization vector, $\gamma$ is the gyromagnetic ratio, $\vec{H}_{\text {eff }}$ is the effective magnetic field, $\vec{h}_{d}$ is the dipole field, and $\vec{m}$ is the dynamic magnetization vector.

To proceed, we linearize $\vec{M}$ and $\vec{H}_{\text {eff }}$ by expanding them into their static and dynamic components,

$$
\begin{aligned}
& \vec{M}=M_{s} \hat{\mathbf{y}}+\vec{m}, \quad|\vec{m}| \ll\left|M_{s}\right|, \\
& \vec{H}_{e f f}=H_{\text {ext }} \hat{\mathbf{y}}+\vec{h}_{e f f}=H_{\text {ext }} \hat{\mathbf{y}}+\vec{h}_{d}+\vec{h}_{s}, \\
& \left|\vec{h}_{\text {eff }}\right| \ll\left|\vec{H}_{\text {ext }}\right|,
\end{aligned}
$$

where $\vec{h}_{s}$ is the microwave magnetic field of a source which excites magnetization precession.

Thus, we rewrite Eq. (1), noting that the second order terms drop out,

$$
\frac{\partial \vec{m}}{\partial t}=-|\gamma|\left(\vec{m} \times H_{e x t} \hat{\mathbf{y}}+M_{s} \hat{\mathbf{y}} \times \vec{h}_{e f f}\right) .
$$

Now, we can carry on and assume that the dynamic variables depend harmonically on time and $\omega$,

$$
\begin{aligned}
\vec{m}(\vec{r}, \omega) & =\vec{m}(\vec{r}) e^{i \omega t}, \\
\vec{h}_{e f f}(\vec{r}, \omega) & =\vec{h}_{e f f}(\vec{r}) e^{i \omega t} .
\end{aligned}
$$

When we solve Eq. (5) with the above assumption, we find that $\vec{m}$ has nonzero components only in the $x$ - and $z$-directions. Solutions to this linearized Landau-Lifshitz equation are well known and involve the Polder microwave susceptibility $\hat{\chi}_{0}$, which relate the dynamic magnetization along the $x$-axis to the effective field at that point, ${ }^{27}$

$$
\vec{m}(x)=\hat{\chi}_{0} \vec{h}_{e f f}(x)
$$

where $\hat{\chi}_{0}$ can be expressed as

$$
\begin{aligned}
& \hat{\chi}_{0}=\left(\begin{array}{cc}
\chi & -i \chi_{a} \\
i \chi_{a} & \chi
\end{array}\right), \quad \chi=\frac{\omega_{H} \omega_{M}}{\omega_{H}^{2}-\omega^{2}}, \\
& \chi_{a}=\frac{\omega \omega_{M}}{\omega_{H}^{2}-\omega^{2}},
\end{aligned}
$$

where $\omega_{H}=\gamma H_{\text {ext }}+i \alpha_{G} \omega, \omega_{M}=4 \pi \gamma M_{s}$, and $\alpha_{G}$ is the Gilbert damping constant. ${ }^{28}$ The addition of the imaginary part to the applied external field is equivalent to taking into consideration the damping loss of the MSSW as it propagates. ${ }^{28}$

To express the dipole field, we average it over the thickness of the film $L$ and we write the average dipole field $h_{d}$ in terms of Green's function $\left(\hat{G}_{d}\right) .{ }^{26}$ Note that this description is only valid for $k L \ll 1$, where $k$ is the MSSW wave number, ${ }^{26}$

$$
\vec{h}_{d}=\int_{-\infty}^{\infty} \hat{G}_{d}\left(x-x^{\prime}\right) \vec{m}\left(x^{\prime}\right) d x^{\prime} .
$$

The precise form for $\hat{G}_{d}$ is not important here. It will be introduced in Subsection II B. What is important is the integral relation between $\vec{h}_{d}$ and $\vec{m}$. This integral dependence reflects the fact that the dipole field of the precessing magnetization is nonlocal. If we use Eqs. (4), (6), and (8), we can see that

$$
\begin{aligned}
\hat{\chi}_{0}^{-1} \vec{m}(x) & =\vec{h}_{d}(x)+\vec{h}_{s}(x) \\
& =\int_{-\infty}^{\infty} \hat{G}_{d}\left(x-x^{\prime}\right) \vec{m}\left(x^{\prime}\right) d x^{\prime}+\vec{h}_{s}(x) .
\end{aligned}
$$

Finally, we can arrive at a solution for $\vec{m}(x)$, which depends on the inverse operator $\hat{G}_{\text {exc }}\left(x-x^{\prime}\right)$,

$$
\vec{m}(x)=\int_{-\infty}^{\infty} \hat{G}_{e x c}\left(x-x^{\prime}\right) \vec{h}_{s}\left(x^{\prime}\right) d x^{\prime},
$$

where $\hat{G}_{e x c}\left(x-x^{\prime}\right)$ satisfies the relation

$$
\int_{-\infty}^{\infty} d x^{\prime}\left(\hat{\chi}_{0}^{-1}-\hat{G}_{d}\left(x-x^{\prime}\right)\right) \hat{G}_{e x c}\left(x-x^{\prime \prime}\right)=\delta\left(x-x^{\prime \prime}\right) .
$$

From the equation above, it is clear that if we can find $\hat{G}_{\text {exc }}$ at every point along the film, we immediately get $\vec{m}$ for a given distribution of $\vec{h}_{s}$. This means that solving for Green's function of excitation is "equivalent" to solving for the dynamic magnetization. Finally note that the integral above is a tensorial one, whereas the integral relevant in the case of BVSW is a scalar one. ${ }^{14}$ This adds complexity to the situation, making MSSW scattering a priori more intricate than BVSW scattering.

\section{B. Solution for Green's function}

We proceed by working in Fourier (reciprocal) space as this greatly decreases computational resources and allows us to use a compact expression for $\hat{G}_{d}$. From our reference frame in reciprocal space, Eq. (8) can be written as

$$
\begin{aligned}
& \vec{h}_{d}(k)=\hat{G}_{d}(k) \vec{m}(k), \\
& \hat{G}_{d}(k)=\left(\begin{array}{cc}
-P(k) & 0 \\
0 & P(k)-1
\end{array}\right),
\end{aligned}
$$

where $P(k)=1+\frac{1}{|k| L}\left(e^{-|k| L}-1\right) .^{29}$

The excitation Green's function has the form

$$
\hat{G}_{e x c}=\left(\begin{array}{ll}
G_{e x c}^{x x} & G_{e x c}^{x z} \\
G_{e x c}^{z x} & G_{e x c}^{z z}
\end{array}\right) .
$$

To obtain the components of Green's function, we need to specify a 1-dimensional point source of a microwave driving field that is compliant with the tensorial form of Green's function. Accordingly, the source field $\vec{h}_{s}$ is modeled as a single frequency (monochromatic) excitation at some point $x=x_{0}$ and with an amplitude $A$. The amplitude of the source is of no importance in this problem; however, it should represent a vector, in accordance with the tensorial character of $\hat{G}_{\text {exc }}$. Modeling the source as the simplest 
possible vector localized at $x=x_{0}$,

$$
\vec{h}_{s}=A \delta\left(x-x_{0}\right) \cdot\left(\begin{array}{l}
1 \\
0
\end{array}\right) \cdot e^{i \omega t}
$$

yields $G_{e x c}^{x x}$ and $G_{e x c}^{z x}$ of Green's function, and modeling the source as

$$
\vec{h}_{s}=A \delta\left(x-x_{0}\right) \cdot\left(\begin{array}{l}
0 \\
1
\end{array}\right) \cdot e^{i \omega t}
$$

allows us to derive the $G_{e x c}^{x z}$ and $G_{e x c}^{z z}$ components. Taking the spatial Fourier transforms of those sources and assuming that $A=1$ and using the information in Eqs. (7), (11), and (12), we find that

$$
\hat{G}_{e x c}(k)=\left(\hat{\chi}_{0}^{-1}-\hat{G}_{d}(k)\right)^{-1} \equiv \hat{Y} / D(\omega, k),
$$

where

$$
\begin{gathered}
\hat{Y}=\left(\begin{array}{cc}
\omega_{H} / \omega_{M}+2-P(k) & -i \omega_{H} / \omega_{M} \\
i \omega_{H} / \omega_{M} & \omega_{H} / \omega_{M}+P(k)
\end{array}\right), \\
D(\omega, k)=\operatorname{det}\left(\hat{\chi}_{0}^{-1}-\hat{G}_{d}(k)\right) .
\end{gathered}
$$

Here, $D(\omega, k)=0$ is the approximate MSSW dispersion relation. ${ }^{29}$ However, as described in the previous literature, ${ }^{30}$ this dispersion relation is nonmonotonic and correctly reproduces the exact Damon-Eschbach dispersion relation ${ }^{27}$ for only $P(k L)<0.5$, which makes the integration in Fourier space inaccurate. Following Ref. 31, we proceed by manually substituting the approximate dispersion relation found in Eq. (16) with the exact Damon-Eschbach dispersion relation, which results in

$$
D_{D E}(\omega, k)=\left(\frac{\omega_{H}}{\omega_{M}}\right)^{2}+\left(\frac{\omega_{H}}{\omega_{M}}\right)+\frac{1}{4}\left(1-e^{-2|k| L}\right)-\left(\frac{\omega}{\omega_{M}}\right)^{2} .
$$

Using Eqs. (10), (16), and (18), we arrive at the result

$$
\vec{m}(k)=\hat{G}_{e x c}(k) e^{-i k x}=\frac{\hat{Y}}{D_{D E}(\omega, k)} e^{-i k x} .
$$

Finally, applying an inverse Fourier transform to Eq. (19) yields

$$
\hat{G}_{e x c}(s)=\frac{1}{2 \pi} \int_{-\infty}^{\infty} \frac{\hat{Y}}{D_{D E}(\omega, k)} e^{i k(s)} d k .
$$

Here, $s=x-x^{\prime}$ and $x^{\prime}$ is the location where a "partial" spin wave is excited and $x$ is the point of observation of the wave of dynamic magnetization excited at point $x^{\prime}$. The "total" amplitude of dynamic magnetization observed at the point $x$ is then a sum (or an integral) over all points $x^{\prime}$ over which the source extends. It is important not to confuse $x^{\prime}$ with $x_{0}, x^{\prime}$ is a dummy coordinate where "some" source is located, while $x_{0}$ is the location of a specific point source that excites a specific spin wave incident on the step. Below, we will see that any $x>x_{\text {step }}$ acts as a source of secondary waves with respect to the incident wave. Hence, any $x>x_{\text {step }}$ can play the role of $x^{\prime}$. Similarly, $x_{0}$ plays the role of $x^{\prime}$ when we calculate the dynamic magnetization of the incident spin wave.

One sees that the expression for Green's function contains an integral over $k$. One way to handle this issue is to evaluate the integral numerically. The second way is to evaluate the integral approximately analytically. In the current work, we will employ the numerical approach, as it is more accurate. However, it is instructive to evoke the analytical solution as the resulting approximate expression gives us an important insight into the physics of the spin wave excitation that remains obscured in the framework of the numerical solution. Therefore, we discuss it briefly below.

In the absence of magnetic losses, the denominator of the kernel of the integral is zero for $k=k_{0}$, where $k_{0}$ satisfies the spin wave dispersion relation $D_{D E}\left(\omega, k_{0}\right)=0$. Obviously, if small magnetic losses are included into the model, the main contribution to the integral will originate from the vicinity of $k_{0}$. Then, one can expand the numerator and the denominator of the kernel of the integral above into Taylor series in the vicinity of $k_{0}$ and only keep the lowest terms of the expansion [the zeroth one for the numerator and the first-order (linear) one for the denominator]. This approximation transforms the integral into a "Cauchy-type" one, which can be analytically evaluated using residues, ${ }^{14}$ leading to

$$
\begin{aligned}
\hat{G}_{e x c}(s)= & \frac{Y\left(\hat{k}_{0}\right)}{2 \pi D_{D E}^{\prime}\left(\omega, k_{0}\right)}\left[2 \pi i e^{i k_{0}^{c}|s|}+e^{i k_{0}^{c}|s|} E_{1}\left(i k_{0}^{c}|s|\right)\right. \\
& \left.+e^{-i k_{0}^{c}|s|} E_{1}\left(-i k_{0}^{c}|s|\right)\right] .
\end{aligned}
$$

Here, $D_{D E}^{\prime}\left(\omega, k_{0}\right)=d D_{D E}(\omega, k) / d k$ evaluated at $k=k_{0}, E_{1}$ is the exponential integral function, and $k_{0}^{c}=k_{0}+i v$, where $v$ is the spatial damping of spin waves due to the presence of magnetic losses in the film. The first term in the square brackets represents two traveling waves excited by the point source in two opposite directions, and the second and the third terms represent the near-field dynamic magnetization of the source. ${ }^{32}$

The main observation that follows from the analytical result above is that the dynamic magnetization field of a source has two components-a far (retarded) field in the form of the two traveling spin waves propagating in two opposite directions from the source and the near (unretarded) field. The latter is strongly localized at the source as the exponential integral function falls steeply with a distance from the origin (in our case in both directions from $s=0$ ). This knowledge will be important to understand the results of our numerical calculations below. As mentioned above, the analytical solution is valid for small magnetic losses, which means that it is applicable when $\hat{Y}(k)$ can be considered as a constant of $k$ and $D_{D E}\left(\omega, k_{0}\right)$ as a linear function of $k$ in the range from $k_{0}-v$ to $k_{0}+v$ or so. This is a good approximation for yttrium iron garnet (YIG) films, and we used it in our previous work, ${ }^{14,32}$ but for metallic ferromagnetic films which are considered in the present paper, this may not always be the case. This is because magnetic damping for these materials can be much higher. Therefore, in the present work, we do not use Eq. (21), for example, calculations of spin wave scattering from the step, but evaluate Green's function numerically using Eq. (20).

\section{SOLUTION TO THE SCATTERING PROBLEM}

We can see that the inverse Polder Susceptibility tensor from Eq. (7) will be different in the region beyond the step (region 1) when compared to the region before the step (region 0). However, $\hat{\chi}$ in region 1 will need to be expressed differently depending on whether the parameter change that characterizes region 1 was a change in external field strength $\Delta H_{\text {ext }}(x)$ or a change in any other 
parameter. In this work, we will deal with the former case only. Other cases may be treated similarly.

Thus, we shall consider the step potential to be characterized by a change in the external field. We can write

$$
\Delta H_{\text {ext }}(x)=A_{s} \theta\left(x-x_{\text {step }}\right),
$$

where $\theta$ denotes the Heaviside step function, $A_{s}$ is the amplitude of the step potential, and $x_{\text {step }}$ is the $x$-coordinate of the step potential. Now, we can simplify $\hat{\chi}_{1}^{-1}$ to write

$$
\begin{aligned}
\hat{\chi}_{1}^{-1}(x) & =\frac{1}{\omega_{M}}\left(\begin{array}{cc}
\omega_{H}+\gamma \Delta H_{\text {ext }}(x) & i \omega \\
-i \omega & \omega_{H}+\gamma \Delta H_{\text {ext }}(x)
\end{array}\right) \\
& =\hat{\chi}_{0}^{-1}+\frac{\gamma}{\omega_{M}} A_{s} \theta(x) \hat{I},
\end{aligned}
$$

where $\hat{I}$ is the diagonal identity tensor. Note that the functions that alter the scattering tensor lie in the diagonal elements when the external field is changed. Now that we have a spatially dependent expression for $\hat{\chi}_{1}^{-1}$, we can proceed as we did in Eq. (9) to find $\vec{m}(x)$

$$
\begin{aligned}
\int_{-\infty}^{\infty}\left(\hat{\chi}_{0}^{-1}-\hat{G}_{d}\left(x-x^{\prime}\right)\right) \vec{m}\left(x^{\prime}\right) d x^{\prime}= & -\frac{\gamma}{\omega_{M}} A_{s} \theta\left(x-x_{\text {step }}\right) \vec{m}(x) \\
& +\delta\left(x-x_{0}\right) \cdot\left(\begin{array}{l}
1 \\
0
\end{array}\right) .
\end{aligned}
$$

Here, we assumed that the dynamic magnetization is driven by a one-dimensional point source located at a point $x_{0}$. Note that as long as the source is located far from the step, the configuration of the microwave driving field of the source does not matter. This is because the dynamic magnetization field incident onto the step will take the form of the eigen-wave of the medium of region 0 , whose form does not depend on the source. The source determines the scalar amplitude of the wave only.

Applying $\hat{G}_{\text {exc }}$, which we found earlier to both sides, we finally arrive at

$$
\vec{m}(x)=-\frac{\gamma}{\omega_{M}} A_{s} \int_{x_{\text {step }}}^{\infty} \hat{G}_{e x c}\left(x-x^{\prime}\right) \vec{m}\left(x^{\prime}\right) d x^{\prime}+\vec{s}(x),
$$

where $\vec{s}(x)=\hat{G}_{e x c} \cdot\left(\begin{array}{l}1 \\ 0\end{array}\right)$. The above equation explains the situation very clearly, so we will discuss it in some detail before we continue. The last term $\vec{s}(x)$ is the original source wave incident onto the step, while the first term is the "correction" to the source wave that region 1 imparts on the MSSW, this is of the form of the Born equation. Obviously, the size of the correction is dependent on the amplitude of the potential difference between regions 0 and 1 , and the correction is built up by adding small contributions from each point in region 1. Therefore, the integral goes from $x_{\text {step }}$ to $\infty$.

We use two approaches to solve the integral equation. The first one is numerical. We proceed by discretizing Eq. (25) into a matrix equation, detailing $\vec{m}(x)$ at each point along the $x$-axis and this will give us a rigorous numerical solution. We also investigate the possibility to obtain a quasianalytical solution of the equation which will be easier to use by experimentalists. To this end, we use the approach of Born approximations for relatively small values of $A_{s}$.

\section{DISCUSSION}

We shall begin by describing how the rigorous numerical solution behaves. Understanding the features of the rigorous numerical solution and the relationships that it satisfies will give us a greater insight into the quasianalytical approach presented later.

\section{A. Rigorous solution}

We used Mathematica to solve the integral in Eq. (25) numerically. We used a discrete mesh with a finite number of equidistant points within a finite range of $x$ values, with the coordinate of the step, $x_{\text {step }}$, located somewhere within this range. The discretization transforms the inhomogeneous integral equation into a vector matrix equation, which is solved using Mathematica's in-built Linear Solve function. The numerical calculation is rather quicktypically, it takes less than a second to complete a simulation run for one value of $A_{s}$.

For the following analysis, the problem is set up such that the source of the MSSW is at $x=0$ and the step occurs at $x_{\text {step }}=2.4 \mu \mathrm{m}$. The amplitude of the step is $A_{s}=-150$ Oe for Fig. 2 and $A_{s}=+140$ Oe for Fig. 3. The parameters entering $\hat{\chi}_{0}$ are $\gamma / 2 \pi=2.82 \times 10^{10} \mathrm{~Hz} / \mathrm{T}=2.82 \times 10^{6} \mathrm{~Hz} / \mathrm{Oe}, H_{\text {ext }}=346 \mathrm{kA} / \mathrm{m}=4350 \mathrm{Oe}$, $\mu_{0} M_{s}=1 \mathrm{~T}$ (which yields $4 \pi M_{s}=10^{4} \mathrm{Oe}$ ), $\omega=23 \mathrm{GHz}, \alpha=0.008$, $L=30 \mathrm{~nm}$. As per the Damon-Eschbach dispersion relation, the values of $H_{\text {ext }}$ and $\omega$ imply that $k=2.959 \mu \mathrm{m}^{-1}$. The length of the film has been discretized into 500 individual points. Figures 2 and 3 show $m_{x}(x)$ using Green's function which we calculated in Eq. (20).

The plots shown in Figs. 2 and 3 are typical and show the main features of the effects of transition of the spin wave through the step and the reflection from the step. The first feature evident in Fig. 2(a) is a sharp exponential decrease in the spin wave amplitude $m_{x}$ with a distance from the source. This happens because magnetic losses in metallic ferromagnetic films are large, and we used a value of the damping parameter characteristic to Permalloy $\left(\mathrm{Ni}_{80} \mathrm{Fe}_{20}\right)$ in this calculation.

Let us now investigate how the MSSW's phase changes with position. In Figs. 2(b) and 3(b), the phase varies linearly in each region. The rate at which the phase changes is different in each region. The slope of the coordinate dependence of the phase is the wave number of the MSSW for the respective area due to the fact that $\frac{\Delta \phi}{\Delta x}=-k$. In Fig. 2(b), we see that the slope becomes steeper beyond the step, which indicates a larger value of $k$ for the same frequency. This intuitively makes sense as the external field in region 1 has been slightly relaxed with respect to region 0 , and the transmitted wave has to adapt its wave number to the new value of the applied static field [see Figs. 1(b) and 1(c)]. Accordingly, in Fig. 3(b) where $A_{s}>0$, we notice that the slope of the phase characteristics becomes much less steep, indicating a lower wave number $k$. This is in agreement with strengthening of the external field in the region beyond the step.

Panels (c) of Figs. 2 and 3 show the ratio $T(x)=\left|m_{x}\left(A_{s}\right)\right|$ $m_{x}\left(A_{s}=0\right) \mid$. The division operation eliminates the effect of losses of the incident wave on the traces from panels (a), which makes the process of reflection and transmission more visible. What one sees in panels (c) is a combination of a wave incident on the step, a wave reflected from it in the negative direction, and a wave transmitted across the step. The location of the field step is shown by a 

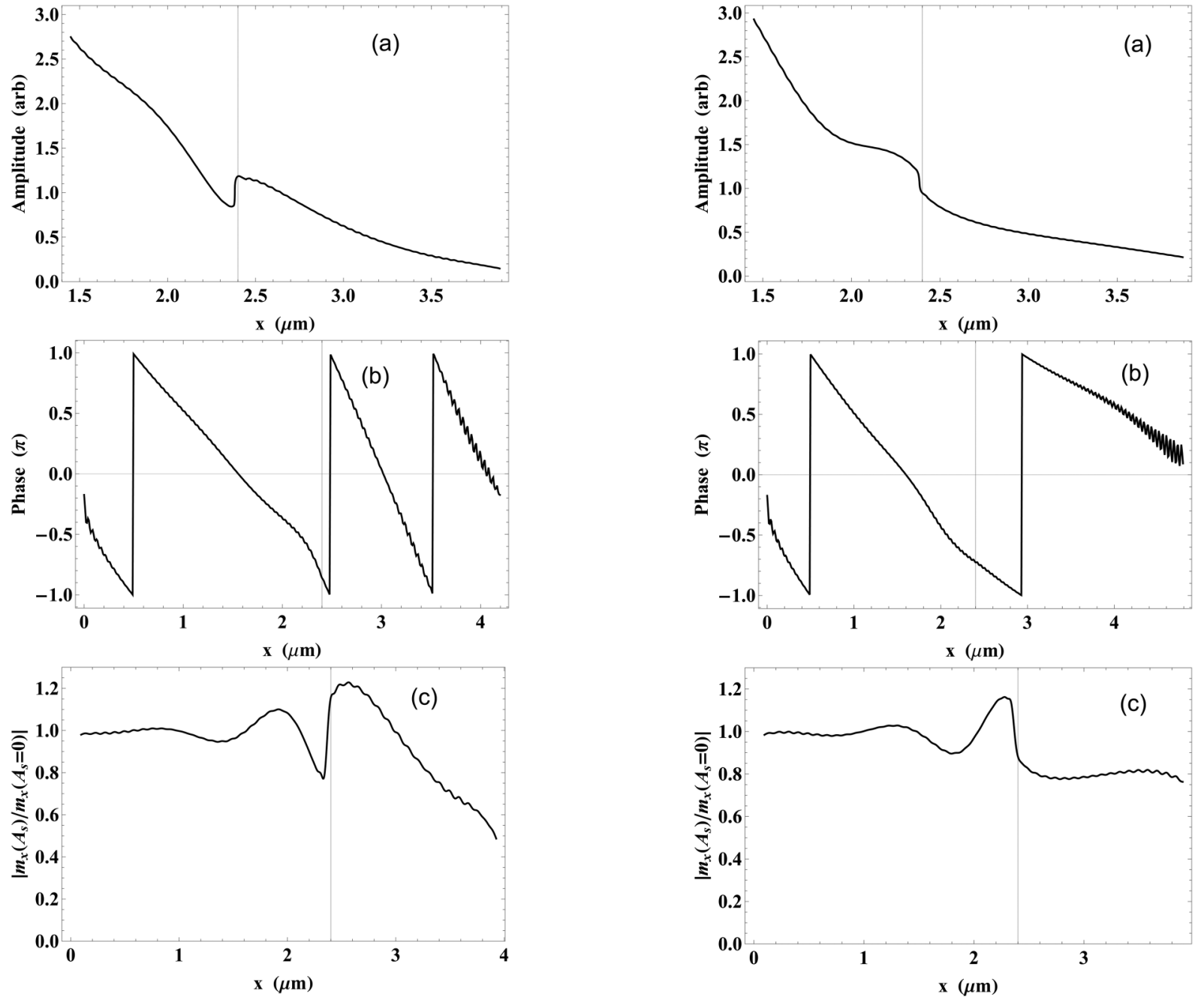

FIG. 2. Rigorous numerical solution of the problem where the step is negative $\left(A_{s}<0\right)$ : amplitude (a), phase (b), and relative amplitude (c) of the $x$ component of the dynamic magnetization vs distance along the plane with a step at $x_{\text {step }}$, denoted by the line. Parameters used are stated at the beginning of Sec. IV A.

vertical line. A first important observation is the oscillating character of $T(x)$ for $x<x_{\text {step }}$. The amplitude of oscillations is maximum at the step and decays exponentially in the negative direction of the $x$-axis. This is evidence that the oscillations are due to formation of a partial standing wave in front of the step. The standing wave pattern arises because of the presence of a wave reflected from the step. The exponential decay of the pattern with the distance is due to damping of the reflected wave-the spatial attenuation of this wave is not eliminated in $T(x)$, but even accentuated, as the amplitude of this wave decays in the opposite direction with respect to the direction of the spatial decay of the incident wave. Similarly, the spatial decay of the wave in region 1 (i.e., beyond the step) is not fully compensated in $T$ in the general case, as the decrement of

the spatial decay for the transmitted wave can be different from that of the incident wave. If this is the case, one will see an exponential drop or rise of the dynamic magnetization amplitude with distance from the step in this region. The decrement/increment of this exponential function will then be equal to the difference in the decrements for the two regions.

One important peculiarity of Fig. 2(a) is the presence of an amplitude jump at $x=x_{\text {step }}$. This implies that the amplitude of the magnetization vector is not conserved at the interface. In other words, the complex amplitude of the transmitted wave is not equal to the sum of the complex amplitudes of the incident and reflected waves. For $A_{s}<0$ [Fig. 2(a)], the step in the amplitude is positive-the wave amplitude rises when it travels past the step.

FIG. 3. Rigorous numerical solution of the problem where the step is positive $\left(A_{s}>0\right)$ : amplitude (a), phase (b), and relative amplitude (c) of the $x$ component of the dynamic magnetization vs distance along the plane with a step at $x_{\text {step }}$, denoted by the vertical line. Parameters used are stated at the beginning of Sec. IV A. 
Note that for $A_{s}<0$, the applied static field is smaller for $x>x_{\text {step }}$ than for $x<x_{\text {step }}$. On the contrary, in the case of Fig. 3(a), $A_{s}>0$, the amplitude of the wave falls sharply after the step potential.

We find that the sign of the jump is in agreement with boundary conditions for dynamic magnetization at $x=x_{\text {step }}$ derived in a previous work, ${ }^{30}$

$$
\left(\begin{array}{l}
m_{x}\left(x=x_{\text {step }}^{+}\right) \\
m_{z}\left(x=x_{\text {step }}^{+}\right)
\end{array}\right)=\hat{M}\left(\begin{array}{l}
m_{x}\left(x=x_{\text {step }}^{-}\right) \\
m_{z}\left(x=x_{\text {step }}^{-}\right)
\end{array}\right),
$$

where

$$
\begin{aligned}
\hat{M}= & \left(\left(\begin{array}{cc}
\chi^{1} & -i \chi_{a}^{1} \\
-i \chi_{a}^{1} & \chi^{1}
\end{array}\right)+\left(\begin{array}{cc}
4 \pi & 0 \\
0 & 0
\end{array}\right)\right)^{-1} \\
& \times\left(\left(\begin{array}{cc}
\chi^{0} & -i \chi_{a}^{0} \\
-i \chi_{a}^{0} & \chi^{0}
\end{array}\right)+\left(\begin{array}{cc}
4 \pi & 0 \\
0 & 0
\end{array}\right)\right)^{-1} .
\end{aligned}
$$

The expression for $\hat{M}$ (27) provides us with an insight into how the amplitude of the MSSW behaves just after the step $\left(x=x_{\text {step }}^{+}\right)$relative to just before the step $\left(x=x_{\text {step }}^{-}\right)$. In a numerical example where $A_{s}=-30 \mathrm{Oe}$, we should see an increase in the amplitude of the wave of approximately $3 \%$, while the rigorous solution of the integral equation exhibits approximately a $5 \%$ increase. Similarly, Eqs. (26) and (27) imply that for $A_{s}=+30$ Oe, the amplitude of the wave should decrease by $3 \%$, while the rigorous solution exhibits a $12 \%$ decrease. The reason for the disparity between the theoretical prediction of Eq. (26) and the rigorous solution is most probably due to the lack of mesh resolution in the locality of the step. However, we notice that the rigorous solution adequately describes the direction of the jump of the amplitude after the step.

Now, we can draw the first conclusion from our theory. A sharp step of film parameters such as effective magnetic field or saturation magnetization results in partial reflection of a spin wave incident on the step. There is a jump of the amplitude of the spin wave at the step, and the sign of the jump depends on the sign of the jump of the parameter which is discontinuous at the step.

As the wave frequency is conserved in the process, the wave number of the wave transmitted through the step has to adapt to the new conditions. It increases or decreases in accordance with the direction of the shift of the local dispersion relation [upward or downward, Figs. 1(b) and 1(c)]. The spin-wave wave number for the region past the step extracted from the slope of the phase characteristics [Figs. 2(b) and 3(b)] is in good agreement with what one expects from the dispersion relation for this area.

It is also important to keep in mind that although the amplitude of dynamic magnetization is discontinuous at the step, the wave energy remains conserved in the process. This follows from the fact that the Poynting vector for the spin-wave scales with its group velocity and the local magnetic energy density associated with the magnetization vector precession. A negative jump of the static field at the step decreases the slope of the dispersion curve for the same frequency and hence the wave group velocity [Fig. 1(c)]. As Fig. 2(a) shows, the spin-wave amplitude jump is positive in this case, which may suggest that the energy is not conserved in our simulation. However, the drop in the group velocity partly compensates for the effect of the increase in the amplitude on the value of the Poynting vector for the transmitted wave. On top of this, the decrease of the applied field decreases the Zeeman energy and modifies the dynamic dipole one for the transmitted wave. All three contributions ensure that the energy is conserved for the three involved waves.

Now, we also want to briefly discuss an artifact which arises in our numerical approach to the solution of the integral equation. In Figs. 2(c) and 3(c), a slight oscillation of the transmitted amplitude is noticeable near the right-hand edge of the plots in the figures. The reason for this waviness is that numerically we cannot extend the bounds of integration in Eq. (25) to $x^{\prime}=\infty$. The integration is only extended to $x=4.8 \mu \mathrm{m}$ in Figs. 2 and 3, which physically means that the region characterized by $\hat{\chi}_{1}$ is not semi-infinite. Due to region 1 not truly extending to positive infinity, there is a reflected wave at the boundary of integration $(x=4.8 \mu \mathrm{m})$, because effectively, the region beyond this point is characterized by $A_{s}=0$. The reflection from this spurious potential step causes interference seen as the above-mentioned waviness in Figs. 2(b) and 3(b). However, if the real length of the simulated potential well/hump is much larger than the free propagation path for spin waves $1 / v$, the wave reflected from the step at $x=4.8 \mu \mathrm{m}$ does not reach $x=x_{\text {step }}$ and hence does not contribute to the amplitude jump at $x=x_{\text {step }}$. Hence, not far from the step, the result is the same as for a well/hump of an infinite length.

\section{B. Transmission coefficient}

We define the transmission coefficient as the proportion of the waves amplitude in the presence of a step potential, compared to the waves amplitude in the absence of any step potential, at some point $x_{o b s}$ past the step

$$
T_{o b s}=\frac{\left|m_{x}\left(x=x_{o b s}\right)\right|}{\left|s_{x}\left(x=x_{o b s}\right)\right|} .
$$

In other words, the transmission coefficient is just a particular point in Figs. 2(c) and 3(c). The choice of the point may be important for future experiments, as there will always be some distance from the step to the location of the spin-wave receiving transducer. Below, we will see that the value of $T$ may be strongly affected by the location of $x=x_{o b s}$. Figure 4 shows the transmission coefficient

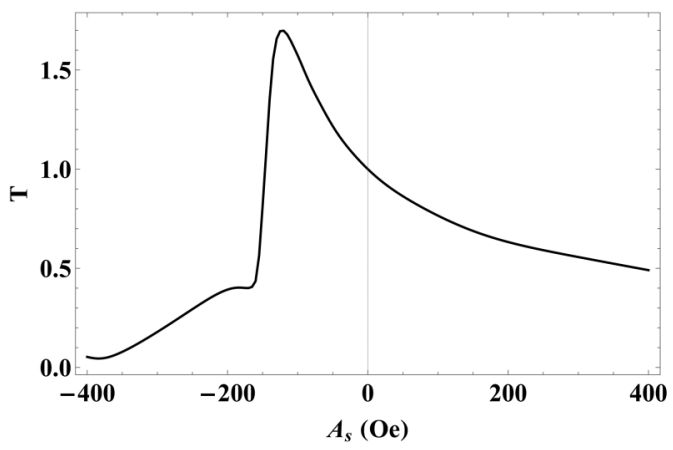

FIG. 4. Relationship between step amplitude and transmission coefficient as given by the rigorous solution for an MSSW, $k=2.959 \mu \mathrm{m}^{-1}$, point at which $T$ was measured was $x=3.93 \times 10^{-7} \mathrm{~m}$ 
for $\omega=23 \mathrm{GHz}$ and $H_{\text {ext }}=4350 \mathrm{Oe}$, which corresponds to a wave number $k_{0}=2.959 \mu \mathrm{m}^{-1}$ for region 0 .

In order to get rid of the influence of the spurious reflection from the far edge of the integration area, we impose a hyperbolic secant "potential" $\left[V(x)=\Delta \alpha \cdot \operatorname{sech}\left(\frac{x-x_{b}}{w}\right)\right]$ of the Gilbert damping parameter in region 1 centered at $x_{b}=4.8 \mu \mathrm{m}$ (its amplitude is 0.002 and width $w=0.2 \mu \mathrm{m}$ ). This has the effect of accelerating the damping of the forward propagating wave before it reaches the end of region 1 and, therefore, not allowing the spurious reflection from the far edge of the integration region to take place. We choose the point $x_{o b s}=3.93 \times 10^{-7} \mathrm{~m}$ as the point of observation of the transmitted amplitude. The observation points lies about $1.4 \mu \mathrm{m}$ from the step, which is possibly the smallest distance from the step achievable in a real-world experiment.

The most important observation from Fig. 4 is that $T$ varies monotonically with $A_{s}$ near $A_{s}=0$. In the region where $A_{s}>0$, we observe a monotonic decrease in the transmission coefficient. The decrease is consistent with formation of a negative jump in Fig. 3(c) at the step. The height of the "fall" increases with an increase in $A_{s}$, which is expected. The region $A_{s}>0$ corresponds to when the dispersion relation shifts up, as shown in Fig. 1(b). Looking closely at Fig. 1(b), it is noted that there are two distinct $A_{s}$ ranges for the positive values, $A_{s}>0$. The first one corresponds to the incident wave frequency sitting within the dispersion curve for $x>x_{\text {step }}$. When $A_{s}>0$, larger $A_{s}$ values correspond to smaller $k_{1}$ values for the transmitted wave. For some $A_{s}$ value, $k_{1}$ drops to zero and past this point, one enters the second $A_{s}$ range, where the frequency of the incident wave falls out of the frequency band for the transmitted wave $\left[\omega\left(k_{0}, H_{\text {ext }}\right)<\omega\left(k_{1}=0, H_{\text {ext }}+\Delta H_{\text {ext }}\right)\right]$. The propagation of the wave is prohibited in region 1 for these $A_{s}$ values. Graphically, this is when the dashed curve in Fig. 1(b) shifts up far enough such that $k_{1}$ no longer exists (more precisely, it becomes imaginary in our solution). If the potential is localized, this is known as the "tunneling regime." The transmission coefficient would gradually fall to 0 with a further increase in $A_{s}$ for a localized potential of the final width. Accordingly, at an infinite distance from the step, the transmission should be zero. However, because we observe the transmission at some finite distance past the step, the behavior is similar to a finite-width potential-a gradual decrease in $T$ is observed with an increase in $A_{s}$. The evanescent wave of magnetization reaches this point with some decreased amplitude with respect to its amplitude at the step. With an increase in $A_{s}$, the amplitude of the evanescent wave drops quicker with distance from the step, which leads to a gradual decrease of $T$ to zero with the increase in $A_{s}$. This is what one will see in a future experiment, while measuring the spin wave amplitude at some distance from the step, as explained above.

Let us now look at the range $A_{s}<0$. For this range in Fig. 4, we observe a monotonic increase in the magnitude of transmission as a function of $\left|A_{s}\right|$ before a sharp decrease at $A_{s}=-125$ Oe. The initial increase is consistent with the formation of a positive dynamic magnetization jump at the step from Fig. 2(c) and boundary condition (26). The growth in the amplitude is very noteworthy. The transmission amplitude for $A_{s}=-125$ Oe is more than 1.5 times larger than for $A_{s}=0$ Oe. Our analysis shows that the reason for this growth is the necessity to satisfy boundary condition (26). Below, we will term this positive contribution to the slope of $T\left(\left|A_{S}\right|\right)$ the "boundary conditions contribution."

The sharp drop in the amplitude for $A_{s}<-125 \mathrm{Oe}$ is not trivial, as the boundary condition predicts that there should be a further growth in the amplitude past the point $A_{s}=-125$ Oe. To provide an explanation for this behavior, we carried out simulations of the amplitude profiles of type [Figs. 2(a) and 3(a)] for different values of the Gilbert damping constant $\alpha$ and for different values of $A_{s}$. These simulations (not shown in the paper) reveal the important role that magnetic damping in the film played in the formation of the maximum in Fig. 4 and the consecutive drop in the transmitted amplitude. As seen from the expression for $T$ earlier, if the decrements of the spatial damping for both regions are the same, the effect of damping does not affect $T$. However, if the decrements are different for the two regions, the magnitude of $T$ will be affected by the "difference" in the decrements. The spatial damping is inversely proportional to the spin wave group velocity. The group velocity is given by the slope of the dispersion curve. Therefore, as long as the slope of the dispersion curves for both regions for a given frequency remains the same, the magnitude of $T$ is not affected by the damping. This happens for small values of $\left|A_{s}\right|$. For larger downshifts of the dispersion curve for the region past the step, the difference in the dispersion curve slopes for the frequency of the incident wave becomes significant. As seen from Fig. 1(c), for $A_{s}<0$ (downshift of the dispersion curve), the slope of the dispersion curve for region 1 actually becomes smaller than that for region 0 . This implies that the group velocity in region 1 drops with respect to region 0 . This leads to larger spatial magnetic damping for region 1 than for region 0 . This difference in damping values is not catered for by the definition of $T$ earlier and gives a negative contribution to the slope of $T\left(\left|A_{s}\right|\right)$. We will call this contribution the "damping contribution."

For small $\left|A_{s}\right|$ values, the positive boundary conditions contribution dominates $T$, and $T$ grows as a function of $A_{s}$. With an approach to the $A_{s}=-125$ Oe mark, the damping contribution starts becoming non-negligible and grows quicker and quicker, as the slope of the dispersion curve decreases significantly with the approach to this point. At $A_{s}=-125 \mathrm{Oe}$, the rate of growth of this contribution with $\left|A_{s}\right|$ becomes equal to the growth rate of the other contribution. As a result, we observe the maximum in the $T\left(\left|A_{s}\right|\right)$ curve. For yet larger $\left|A_{s}\right|$ values, the negative damping contribution starts to grow quicker than the "boundary conditions" one and the transmission starts to drop.

This explanation is confirmed by our simulations taken at different distances from the step. For smaller distances from the step, the maximum in the $T\left(\left|A_{s}\right|\right)$ dependence moves to larger $\left|A_{s}\right|$ values and its height increases. Very close to the step, $T$ keeps growing within the whole range of observed $\left|A_{s}\right|$ values. As we already mentioned several times, in real-world experiments, there will be some distance between the step and the point of measurement of the transmission coefficient. Therefore, in the real experimental situation, the nonmonotonic $T\left(\left|A_{s}\right|\right)$ dependence as in Fig. 4 will be observed, and the experimentalists will need to keep in mind that the negative slope of the dependence for large $\left|A_{s}\right|$ values is just a kind of artifact. 


\section{Born approximations}

The last point that we would like to quickly discuss in this article is whether it is possible to obtain a quasianalytical solution for the integral equation and hence simplify the calculation of $T$. To this end, we investigate the approach of Born approximations.

The first Born approximation on the system is obtained by letting $\vec{m}(x)$ in the integral to be equal $\vec{s}(x)$. This leads us to

$$
\vec{m}^{(1)}(x)=-\frac{\gamma}{\omega_{M}} A_{s} \int_{x_{\text {step }}}^{\infty} \hat{G}_{e x c}\left(x-x^{\prime}\right) \vec{s}\left(x^{\prime}\right) d x^{\prime}+\vec{s}(x),
$$

where $\vec{m}^{(1)}(x)$ is the sought vector amplitude of the dynamic magnetization. The second Born approximation is then obtained by substituting the solution above back into the integral equation. This yields

$$
\vec{m}^{(2)}(x)=-\frac{\gamma}{\omega_{M}} A_{s} \int_{x_{\text {step }}}^{\infty} \hat{G}_{e x c}\left(x-x^{\prime}\right) \vec{m}^{(1)}\left(x^{\prime}\right) d x^{\prime}+\vec{s}(x) .
$$
the form

If we put Eq. (29) into Eq. (30), we can express $\vec{m}^{(2)}(x)$ in

$$
\vec{m}^{(2)}(x)=\left(\frac{\gamma}{\omega_{M}} A_{s}\right)^{2} B_{2}-\left(\frac{\gamma}{\omega_{M}} A_{s}\right) B_{1}+\vec{s}(x) .
$$

where

$$
\begin{aligned}
& B_{1}=\int_{x_{\text {step }}}^{\infty} \hat{G}_{\text {exc }}\left(x-x^{\prime}\right) \vec{s}\left(x^{\prime}\right) d x^{\prime}, \\
& B_{2}=\int_{x_{\text {step }}}^{\infty} \hat{G}_{e x c}\left(x-x^{\prime}\right) \int_{x_{\text {step }}}^{\infty} \hat{G}_{e x c}\left(x^{\prime}-x^{\prime \prime}\right) \vec{s}\left(x^{\prime \prime}\right) d x^{\prime \prime} d x^{\prime} .
\end{aligned}
$$

One sees that the first Born approximation (which scales with $B_{1}$ ) is linear in $A_{s}$, and the second Born approximation (which scales with $B_{2}$ ) gives a correction of the second order in $A_{s}$.

There are two ways to use the expressions above. The first one is by employing the approximate Green's function in Eq. (20) for the calculation of the integrals. Here, one has to note that because the expression for Green's function contains special functions, the integrals cannot be evaluated analytically in an easy way. Therefore, numerical evaluation of integrals is needed. This is not a big problem, and this approach may be more convenient for experimentalists who will wish to compare our theory with their experimental data.

In this work, we use a different approach though. We employ the same rigorous numerical Green's function and calculate all the integrals numerically. Although no analytical solution is obtained within either approach, there is a significant improvement with respect to the direct numerical solution of the integral equation carried out above. This improvement is the simplicity of numerical evaluation of the integrals in (32) with respect to the direct numerical solution of the integral equation. Basically, the numerical evaluation of the integrals can be reduced to just one line in MathCAD or Mathematica.

From Fig. 5, one sees that the second Born approximation provides a reasonably accurate representation of the transmission coefficient of the rigorous solution for small changes in the external

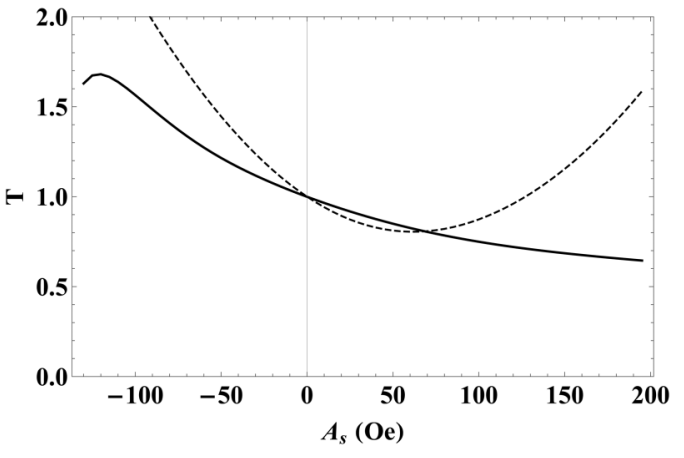

FIG. 5. Potential due to $\Delta H_{\text {ext }}$. relationship between step amplitude and transmission coefficient as given by the rigorous solution for an MSSW (solid line) and the second Born approximation (dashed line), $k=2.959 \mu \mathrm{m}^{-1}$, point at which $T$ was measured was $x=3.93 \times 10^{-7} \mathrm{~m}$.

field amplitude $(-10 \mathrm{Oe}<\mathrm{As}<100 \mathrm{Oe})$. This confirms that it is possible to obtain a simple solution for the integral equation valid for smaller values of $A_{s}$.

\section{CONCLUSION}

A theoretical analysis of MSSW propagation in thin ferromagnetic films in the presence of step potentials characterized by changes in external field strength was conducted. A quasianalytical approach was arrived at that allows us to describe the contribution that a potential imparts on the amplitude and phase of a MSSW. The formalism takes into account the Zeeman and dipole-dipole interactions in the medium but does not account for the inhomogeneous exchange interaction in it. Therefore, our results are valid for spin wave wavelengths, which are much larger than the exchange length for the material. The result of our derivation is an integral equation for the vector amplitude of dynamic magnetization. The equation is solved numerically in order to investigate spin wave transmission through the step potential.

We found that a spin wave incident onto the step is partially reflected and partially transmitted. At the step, the sum of the complex-valued amplitudes of the incident and reflected waves is not equal to the amplitude of the transmitted wave. Accordingly, a jump of the magnetization precession amplitude takes place at the step. The sign of the jump depends on the sign of the step potential $A_{s}$. A positive $A_{s}$ (which corresponds to an increase in the static field at the step) leads to a negative jump (a decrease) of the magnetization precession amplitude at the step and vice versa. Furthermore, while traveling past the step, the spin wave has to adjust its wave number to a new value, consistent with the spin wave dispersion law for the magnetic parameters characteristic to the area past the step. This leads to a particular phase characteristic seen in the numerical simulation.

The efficiency of spin wave transmission through the step was analyzed as a function of $A_{s}$. In order to quantify the efficiency, we introduced the notion of the transmission coefficient. It characterizes the complex-valued amplitude of the transmitted wave relative to the amplitude of the incident wave. We found that the transmission coefficient is a monotonically falling function of $A_{s}$ in a broad 
range of negative and positive $A_{s}$ values. However, the presence of magnetic losses in the material leads to a change of the character of this dependence from falling to rising at some negative value of $A_{s}$ (for which the transmission coefficient is maximum). The position of the maximum depends on the magnetic loss parameter for the film and the distance from the step at which the transmission coefficient is observed.

In our work, we considered infinitely sharp potential steps. As follows from Ref. 14, smoother steps, where the potential varies on the length scale of the wavelength of the incident spin wave, may produce smaller reflection and better transmission from the step. The limit of the slowly varying potential was outside the scope of our work whose aim was to find a way to efficiently control the amplitude of the signal transmitted through a potential step.

We also showed that a quasianalytical solution in the form of the second Born approximation is valid for a relatively large range of $A_{s}$ values. The solution takes the form of a sum of simple integrals, which can be easily evaluated numerically, and the evaluation is instantaneous with a modern PC. This represents an important technical improvement with respect to the direct numerical solution of the initial integral equation.

\section{ACKNOWLEDGMENTS}

A Research Collaboration Award from the University of Western Australia and the grant Magmatch (Labex NIE No. 11-LABX-0058) from the Agence Nationale de la Recherche, France, are acknowledged.

\section{REFERENCES}

${ }^{1}$ C. G. Sykes, J. D. Adam, and J. H. Collins, Appl. Phys. Lett. 29, 339 (1976).

${ }^{2}$ J. P. Parekh and H. S. Tuan, IEEE Trans. Magn. 13, 1246 (1977).

3. P. Parekh and H. S. Tuan, Appl. Phys. Lett. 31, 709 (1977).

${ }^{4}$ J. P. Parekh and H. S. Tuan, IEEE Trans. Microw. Theory Tech. 26, 1039 (1978).

5. P. Parekh and H. S. Tuan, Appl. Phys. Lett. 33, 267 (1978).

${ }^{6}$ S. R. Seshadri, IEEE Trans. Microw. Theory Tech. 26, 1039 (1978).

${ }^{7}$ Yu. V. Gulyaev and S. A. Nikitov, Sov. Phys. Solid State 26, 3678 (1981).

${ }^{8}$ Yu. Gulyaev, S. A. Nikitov, and V. P. Plesskii, Tech. Phys. 52, 799 (1982).

${ }^{9}$ G. A. Vugal'ter and I. A. Gilinski, Radiophys. Quantum Electron. 32, 869 (1989).

${ }^{10}$ J. M. Owens, C. V. Smith, Jr., S. N. Lee, and J. H. Collins, IEEE Trans. Magn. 14, 820 (1978).
${ }^{11}$ A. V. Voronenko and S. V. Gerus, Tech. Phys. 10, 746 (1984).

${ }^{12}$ R. L. Carter, J. M. Owens, C. V. Smith, Jr., and K. W. Reed, J. Appl. Phys. 53, 2655 (1982).

${ }^{13}$ S. O. Demokritov, A. A. Serga, A. Andre, V. E. Demidov, M. P. Kostylev, B. Hillebrands, and A. N. Slavin, Phys. Rev. Lett. 93, 047201 (2004).

${ }^{14}$ M. P. Kostylev, A. A. Serga, T. Schneider, T. Neumann, B. Leven, B. Hillebrands, and R. L. Stamps, Phys. Rev. B 76, 184419 (2007).

${ }^{15}$ T. Schneider, A. A. Serga, A. V. Chumak, B. Hillebrands, and M. P. Kostylev, Europhys. Lett. 930, 27003 (2010).

${ }^{16}$ A. V. Chumak, A. A. Serga, S. Wolff, B. Hillebrands, and M. P. Kostylev, Appl. Phys. Lett. 94, 172511 (2009).

${ }^{17}$ T. Neumann, A. A. Serga, B. Hillebrands, and M. P. Kostylev, Appl. Phys. Lett. 94, 042503 (2009).

${ }^{18}$ A. V. Chumak, T. Neumann, A. A. Serga, B. Hillebrands, and M. P. Kostylev, J. Phys. D 42, 205005 (2009).

${ }^{19}$ A. V. Chumak, V. I. Vasyuchka, A. A. Serga, M. P. Kostylev, V. S. Tiberkevich, and B. Hillebrands, Phys. Rev. Lett. 108, 257207 (2012).

${ }^{20}$ M. P. Kostylev, A. A. Serga, T. Schneider, B. Leven, and B. Hillebrands, Appl. Phys. Lett. 87, 153501 (2005).

${ }^{21}$ T. Schneider, A. A. Serga, B. Hillebrands, M. P. Kostylev, and J. Nanoel, Optoel. 3, 69 (2008).

${ }^{22}$ N. Tahir, R. Gieniusz, A. Maziewski, R. Bali, M. P. Kostylev, S. Wintz, H. Schultheiss, S. Facsko, K. Potzger, J. Lindner, and J. Fassbender, IEEE Trans. Magn. 50, 6101304 (2014).

${ }^{23}$ G. Shimon, C. A. Ross, and A. O. Adeyeye, J. Appl. Phys. 118, 153901 (2015).

${ }^{24}$ J.-H. Moon, S.-M. Seo, K.-J. Lee, K.-W. Kim, J. Ryu, H.-W. Lee, R. D. McMichael, and M. D. Stiles, Phys. Rev. B 88, 184404 (2013).

${ }^{25}$ C. Bayer, M. P. Kostylev, and B. Hillebrands, Appl. Phys. Lett. 88, 112504 (2006).

${ }^{\mathbf{2 6}}$ T. Schneider, A. A. Serga, T. Neumann, B. Hillebrands, and M. P. Kostylev, Phys. Rev. B 77, 214411 (2008).

${ }^{27}$ D. Stancil and A. Prabhakar, Spin Waves: Theory and Applications (Springer, 2009), ISBN 978-0-387-77865-5.

${ }^{28}$ A. G. Gurevich and G. A. Melkov, Magnetization Oscillations and Waves (CRC Press, 1996), ISBN 9780849394607.

${ }^{29}$ B. Kalinikos, Russ. Phys. J. 24, 718 (2004).

${ }^{30}$ M. P. Kostylev and N. A. Sergeeva "Collective and individual modes on onedimensional bi-layered magnetic structures" in Magnetic Properties of Laterally Confined Nanometric Structures, edited by G. Gubbiotti (Transworld Research Network, 2009), ISBN: 81-7895-212-2.

${ }^{31}$ J. Hutomo, P. Metaxas, and M. Kostylev, "Scattering of magnetostatic surface waves in ferromagnetic films from localized inhomogeneities" (unpubished).

${ }^{32}$ C. S. Chang, M. Kostylev, E. Ivanov, J. Ding, and A. O. Adeyeye, Appl. Phys. Lett. 104, 032408 (2014). 